\title{
ALBI and Child-Pugh score in predicting mortality in chronic liver disease patients secondary to alcohol - A retrospective comparative study
}

\author{
Nagaraja B S ${ }^{1}$, Madhumathi $\mathbf{R}^{2}$, Sanjeet S B ${ }^{3}$, Umesh K J ${ }^{4}$, Nandish Kumar $\mathbf{S}^{5}$ \\ ${ }^{1}$ Professor and Head, ${ }^{2}$ Professor, ${ }^{3,4}$ Post-Graduate, Department of General Medicine, Bangalore Medical College and \\ Research Institute, ${ }^{5}$ Post-Graduate Department of Community Medicine, Bangalore Medical College and Research \\ Institute, KR Road, Bangalore, Karnataka, India
}



\section{A B S T R A C T}

Background: The severity of liver dysfunction in chronic liver disease is often estimated with Child-Pugh (CTP) classification or model for end-stage liver disease (MELD) score. The albumin-bilirubin (ALBI) score is a new model for assessing the severity of liver dysfunction, which is simple and more objective. Aims and Objective: The present study was aimed to retrospectively compare the performance of ALBI score with Child-Pugh score for predicting the mortality in patients with chronic liver disease. Materials and Methods: Data of patients with chronic Liver disease irrespective of etiology were retrospectively reviewed. Child Pugh score and ALBI score were calculated for the patients and results from ROC curves were analysed. Results: Study conducted on 299 patients of chronic liver disease, age distribution was between $20-85$ years with mean age of patients being $45.7+/-10.94$ years, sex ratio male: female is $265: 34$ with mortality rate of $19.73 \%$. The area under curves of ROC of ALBI and Child pugh are 0.586 and 0.549 respectively. Conclusion: Ability of ALBI score for predicting mortality was comparable with that of Child Pugh score but Child pugh score of more than 10 has got better performance of predicting mortality as compared to ALBI score.

\section{Access this article online}

Website:

http://nepjol.info/index.php/AJMS DOI: 10.3126/ajms.v10i3.23171 E-ISSN: 2091-0576 P-ISSN: 2467-9100

Key Words: ALBI; Mortality; CLD; Child-pugh

\section{INTRODUCTION}

WHO estimates 2 billion people as consuming alcohol and 76.3 million have alcohol use disorders. Thirty percent of Indian adults use alcohol among which $4-13 \%$ is daily consumers. The alcohol consumption has gone up by $30 \%$ in 2015. Estimate of 14 million has been made as heavy consumers. Looking at this data the burden of alcoholic liver disease on community is obvious. Alcohol abuse leads to spectrum of liver diseases ranging from fatty liver, alcoholic hepatitis to cirrhosis and hepatocellular carcinoma. Liver cirrhosis is a common cause of death in the world. ${ }^{1,2}$ The accurate prognostication of liver cirrhosis is important in our daily practice. The most commonly used tool to predict the prognosis of liver cirrhosis is Child-Pugh score. ${ }^{3}$ However, it has been established for a long time, and its components are selected primarily based on the surgeons' experiences. Model for end-stage liver disease (MELD) score is another tool for prognostic assessment of liver cirrhosis. ${ }^{4,5}$ Until now, there are lots of controversy regarding the comparison of Child-Pugh versus MELD scores. ${ }^{6-9}$ More recently, albumin-to-bilirubin (ALBI) score has been proposed as a novel, simple, and readily available model calculated using mathematical formula $-0.085 \mathrm{X}$ (alb g/l) $+0.66 \mathrm{X} \log (\mathrm{bilumol} / \mathrm{l})$.

The albumin-bilirubin (ALBI) score, by combining the serum albumin and bilirubin, is a new model for assessing the severity of liver dysfunction. Recently, Johnson and colleagues reported that the ALBI score more accurately 
predicts patients' mortality without requiring subjective determinants of liver failure, including ascites and encephalopathy, in patients with hepatocellular carcinoma. ${ }^{10}$ A retrospective study also investigated the prognostic significance of the ALBI score among patients with primary biliary cirrhosis. ${ }^{11}$ It is found that the ALBI score seems to outperform other scores (such Child-Pugh and MELD score) for predicting the occurrence of hepatic events in such patients. Furthermore, Chen et $\mathrm{al}^{12}$ demonstrated that ALBI score had a significantly better performance for long-term survival prediction in patients with HBV-related cirrhosis than the Child-Pugh or MELD scores. Herein, we attempted to study the ALBI score for in-hospital death in Alcoholic cirrhotic patients.

\section{Aims and Objectives}

- To calculate ALBI and Child Pugh Score in CLD patients secondary to alcohol

- To assess the ALBI in predicting the mortality in CLD patients secondary to alcohol

- To evaluate the discriminative abilities ALBI and Child Pugh score in predicting the in-hospital mortality in CLD patients secondary to alcohol.

\section{MATERIALS AND METHODS}

\section{Study design}

The study was conducted at Bowring and Lady Curzon Hospital (attached to Bangalore Medical College and Research Institute). Cirrhotic patients secondary to alcohol admitted in the hospital between January 2017 and December 2017 were retrospectively reviewed and the data of the patients were collected. Approval was obtained from the institutional ethical committee.

\section{Inclusion criteria}

- Age $>18$ years

- Liver cirrhosis patients secondary to alcohol.

\section{Exclusion criteria}

- CLD due to HBV, HCV, malignancy, metabolic causes, autoimmune hepatitis.

\section{Method of collection of Data}

Detailed history and clinical examination was done for all patients. Routine investigations like complete hemogram, renal function test, liver function test, serum electrolytes, HIV, HBsAG, HCV, PT/INR, APTT, USG abdomen and other relevant investigations were noted. Diagnosis of liver cirrhosis was established by USG abdomen with shrunken liver with altered echo texture.

ALBI score and Child Pugh score were calculated and compared.
ALBI score $=[-0.085 \mathrm{X}(\mathrm{alb} g / \mathrm{l})+0.66 \mathrm{X} \log (\mathrm{bilumol} / \mathrm{l})]$

\section{Method of statistical analysis}

All statistical analysis were performed using Medcalc software. Continuous data were expressed as the mean +/- SD and median with minimum and maximum. Categorical data were expressed as the frequency. ROC curve were performed to identify the discriminative ability of ALBI and Child Pugh score in predicting in-hospital mortality. AUC were calculated and compared. The best cut off value was selected as the sum of sensitivity and specificity was maximal. The sensitivity, specificity, positive likelihood ratio, negative likelihood ratio were reported.

\section{RESULTS}

The sample size in our study was 299 patients. The age distribution was between 20-85 years with mean age of patient being $45.7+/-10.94$ years. Two-hundred sixty five were males and 34 were females. Among 299 patients, 59 patients had in-hospital mortality and 240 were discharged with mortality percentage of $19.73 \%$.

Comparison of in-hospital mortality with ALBI and Child PUGH scores

The in hospital morality was $19.73 \%$. The Area under curve (AUC) of the ALBI score for predicting the in-hospital mortality was 0.586 (confidence interval: 95\%: 0.528 -0642) (Figure 1). The best cut-off value of -1.01 , with sensitivity of $94.92 \%$, a specificity of $32.5 \%$, positive likelihood ratio (PLR) of 1.406 and negative likelihood ratio (NLR) 0.156.

The AUC of the Child Pugh score for predicting the inhospital mortality was 0.549 (confidence interval 95\%: 0.490-0.606) (Figure 2). The Best cut-off value of the Child-Pugh score was 10, with a sensitivity of $76.27 \%$, a specificity of $34.58 \%$, PLR of 1.165 and NLR of 0.686 .

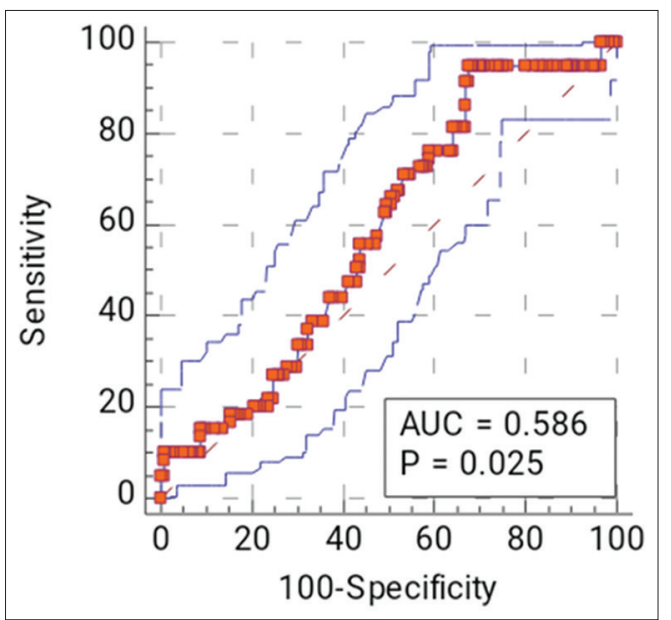

Figure 1: ROC curve of ALBI score for predicting In Hospital Mortality 
The AUC for predicting the in-hospital mortality was not significantly different between the Child Pugh and ALBI scores. (Child pugh and ALBI: $\mathrm{P}=0.4461$ ). (Table 1 and Figure 3)

Thus the performance of Child Pugh score is higher than ALBI score. (Table 2)

\section{DISCUSSION}

Child pugh score and MELD score have been studied extensively for their prognostic abilities and have shown to perform good to predict the mortality of cirrhotic patients. But the cumbersome calculation of scores and the variables included in them have subjective variability has led to the development of ALBI score.

ALBI score involves only two variables and already studied in various liver disorders such as HBV, HCC, primary

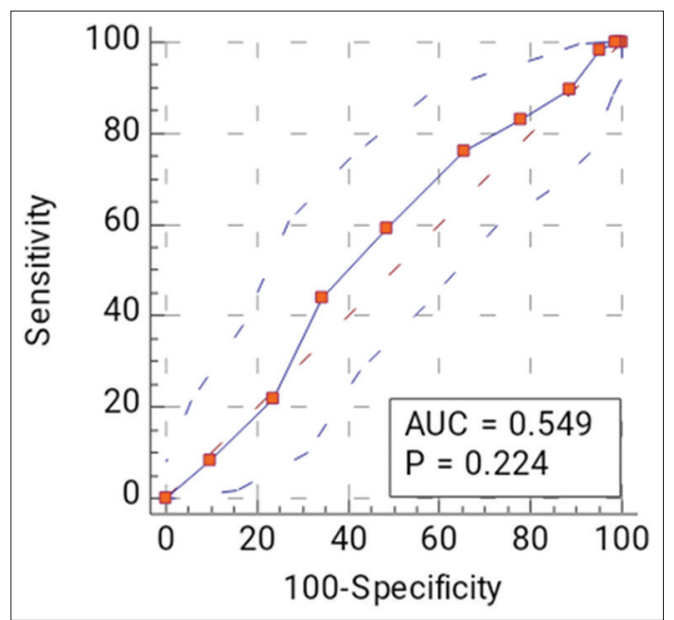

Figure 2: ROC curve of child Pugh score for predicting In Hospital Mortality

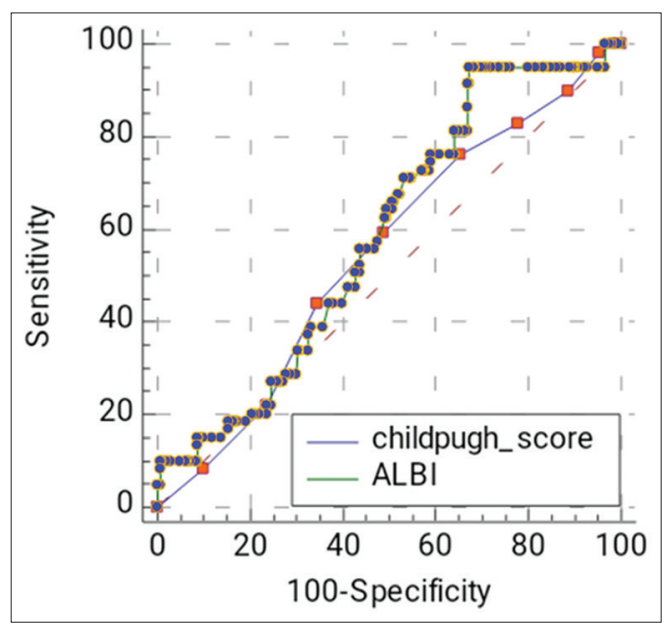

Figure 3: Graph showing comparison of ROC curves biliary cirrhosis and has shown to perform good and is comparable with the Child pugh and MELD scores.

In our study attempt has been made to compare the discriminative ability of ALBI score with that of the Child pugh score in predicting the in-hospital mortality in alcoholic cirrhosis patients. ALBI score showed better performance compared to Child pugh score in predicting mortality but there was no statistical difference between them.

In a study by Lichun Shao et al, ALBI score has demonstrated similar ability as that of Child pugh and MELD score in predicting in-hospital mortality in cirrhosis. It also suggests that ALBI score can be readily used as prognostic model. ${ }^{13}$ Another study by Bo Chen, et al showed that the ALBI score determined on admission indicates the likelihood of survival of an CLF patients. ${ }^{14}$

In a study conducted by Deli Zou et al, ALBI score had the largest AUC, followed by MELD score and Child Pugh scores in ROC curves, so they concluded that ALBI score has moderate to high prognostic performance. ${ }^{15}$

A study conducted by Peng et al, showed there was no significant difference in predicting in-hospital mortality in cirrhotic patients. ${ }^{16}$

A retrospective Study done by Xavier SA et al, on 111 patients between Jan 2011 and Nov 2015, came out with conclusion that ALBI score is particularly useful in the assessment of short come outcomes, with a better performance than the most commonly used scores. ${ }^{17}$

\section{Limitations of the study}

The limitations of our study were that it was a retrospective study, the late mortality was not considered and follow up was not done.

\begin{tabular}{lccc}
\multicolumn{4}{l}{ Table 1: Comparison of ROC curves } \\
\hline Variable & AUC & SE & $\mathbf{9 5 \%} \mathbf{~ C l}$ \\
\hline Child pugh score & 0.549 & 0.0401 & 0.490 to 0.606 \\
ALBI & 0.586 & 0.0382 & 0.528 to 0.642 \\
\hline
\end{tabular}

\begin{tabular}{|c|c|}
\hline Child pugh score ALBI & Values \\
\hline Difference between areas & 0.0370 \\
\hline Standard Error & 0.0486 \\
\hline $\begin{array}{l}95 \% \text { Confidence Interval } \\
\text { z statistic }\end{array}$ & $\begin{array}{c}-0.0582 \text { to } 0.132 \\
0.762\end{array}$ \\
\hline Significance level & $P=0.4461$ \\
\hline
\end{tabular}




\section{CONCLUSION}

AUC of the ALBI score and the Child pugh score were comparable and there was no statistical difference between them. ALBI can be used in place of Child pugh score in peripheral centres to assess the prognosis of CLD patients secondary to alcohol in view of simple calculation, only two variables and no subjective variation of the score.

\section{REFERENCES}

1. Blachier M, Leleu H, Peck-Radosavljevic M, Valla D-C and Roudot-Thoraval F. The burden of liver disease in Europe: A review of available epidemiological data. Journal of Hepatology 2013; 58(3):593-608.

2. Lozano R, Naghavi M, Foreman K, Lim S, Shibuya K, Aboyans V, et al. Global and regional mortality from 235 causes of death for 20 age groups in 1990 and 2010: a systematic analysis for the Global Burden of Disease Study 2010. Lancet 2012; 380:20952128.

3. Pugh RNH, Murray-Lyon IM, Dawson JL, Pietroni MC and Williams R. Transection of the oesophagus for bleeding oesophageal varices. British Journal of Surgery 1973; 60(8):646-649.

4. Kamath $P$, Wiesner R, Malinchoc M, Kremers W, Therneau T, Damico G, et al. A model to predict survival in patients with endstage liver disease. Gastroenterology 2001; 120(5).

5. Kamath PS and Kim WR. The model for end-stage liver disease (MELD). Hepatology 2007;45(3):797-805.

6. Gheorghe L, lacob S, Simionov I, Vandan R, Gheoghe C, lacob R, et al. Natural history of compensated viral B and D cirrhosis. Rom J Gastroenterol 2005; 14:329-335.

7. Cholongitas E, Marelli L, Shusang V, Senzolo M, Rolles K, Patch D, et al. A systematic review of the performance of the model for end-stage liver disease (MELD) in the setting of liver transplantation. Liver Transplantation 2006; 12(7):1049-1061.

8. Peng Y, Qi X, Dai J, Li H and Guo X. Child-Pugh versus MELD score for predicting the in-hospital mortality of acute upper gastrointestinal bleeding in liver cirrhosis. Int J Clin Exp Med 2015; 8:751-757.

9. Chalasani N, Kahi C, Francois F, Pinto A, Marathe A, Bini EJ, et al. Model for end-stage liver disease(MELD) for predicting mortality in patients with acute variceal bleeding. Hepatology 2002; 35(5):1282-1284.

10. Johnson PJ, Berhane S, Kagebayashi C, Satomura S, Teng M, Reeves HL, et al. Assessment of Liver Function in Patients With Hepatocellular Carcinoma: A New EvidenceBased Approach-The ALBI Grade. Journal of Clinical Oncology 2015; 33(6):550-558.

11. Chan AWH, Chan RCK, Wong GLH, Wong VWS, Choi PCL, Chan HLY, et al. New simple prognostic score for primary biliary cirrhosis: Albumin-bilirubin score. Journal of Gastroenterology and Hepatology 2015; 30(9):1391-1396.

12. Chen R-C, Cai Y-J, Wu J-M, Wang X-D, Song M, Wang Y-Q, et al. Usefulness of albumin-bilirubin grade for evaluation of long-term prognosis for hepatitis B-related cirrhosis. Journal of Viral Hepatitis 2016; 24(3):238-245.

13. Shao L, Han B, An S, Ma J, Guo X, Romeiro FG, et al. Albuminto-bilirubin score for assessing the in-hospital death in cirrhosis. Translational Gastroenterology and Hepatology 2017;88.

14. Chen B and Lin S. Albumin-bilirubin (ALBI) score at admission predicts possible outcomes in patients with acute-on-chronic liver failure. Medicine 2017;96(24).

15. Zou D, Qi X, Zhu C, Ning Z, Hou F, Zhao J, et al. Albumin-bilirubin score for predicting the in-hospital mortality of acute upper gastrointestinal bleeding in liver cirrhosis: A retrospective study. The Turkish Journal of Gastroenterology 2016; 27(2):180-186.

16. Peng Y, QiX, Tang S, Deng H, Li J, Ning Z, etal. Child-Pugh, MELD, and ALBI scores for predicting the in-hospital mortality in cirrhotic patients with acute-on-chronic liver failure. Expert Review of Gastroenterology \& Hepatology 2016; 1-10.

17. Xavier SA, Vilas-Boas R, Carvalho PB, Magalhães JT, Marinho $\mathrm{CM}$ and Cotter JB. Assessment of prognostic performance of Albumin-Bilirubin, Child-Pugh, and Model for End-stage Liver Disease scores in patients with liver cirrhosis complicated with acute upper gastrointestinal bleeding. European Journal of Gastroenterology \& Hepatology 2018;1.

\footnotetext{
Authors Contribution:

NBS-Concept and Design of the study, Manuscript preparation, Statistically analysed and interpreted, Critical revision of the Manuscript; MR-Concept and Design of the study, Statistically analysed and interpreted, Critical revision of the manuscript; SSB- Collected Data, Statistically analysed and interpreted, helped in preparing draft of manuscript; UKJ-Reviewed the literature, helped in preparing first draft of Manuscript, collected Data; NKS- Assisted in statistical analysis.

Work attributed to:

Department of General Medicine, Hospitals attached to Bangalore Medical College and Research Institute.

Orcid ID:

Dr. Nagaraja B S- (i) https://orcid.org/0000-0002-7750-697X

Dr. Madhumathi R - (D) https://orcid.org/0000-0002-7534-1059

Dr. Sanjeet S B - (10) https://orcid.org/0000-0002-2243-9917

Dr. Umesh K J - (1) https://orcid.org/0000-0002-1816-9125

Dr. Nandish Kumar S- (1) https://orcid.org/0000-0002-8978-8644

Source of Support: Bangalore Medical College and Research Institute fund, Conflict of Interest: None.
} 